\title{
Modified Hadamard product properties of certain class of analytic functions with varying arguments defined by Sălăgean and Ruscheweyh derivative
}

\author{
Ágnes Orsolya Páll-Szabó
}

\begin{abstract}
In this paper we study the modified Hadamard product properties of certain class of analytic functions with varying arguments defined by Sălăgean and Ruscheweyh derivative. The obtained results are sharp and they improve known results.
\end{abstract}

Mathematics Subject Classification (2010): 30C45.

Keywords: Analytic functions, modified Hadamard product, Sălăgean and Ruscheweyh derivative.

\section{Introduction}

Let $\mathcal{A}$ denote the class of functions of the form:

$$
f(z)=z+\sum_{k=2}^{\infty} a_{k} z^{k},
$$

which are analytic in the open unit $\operatorname{disc} U=\{z \in \mathbb{C}:|z|<1\}$. Let $g \in \mathcal{A}$ where

$$
g(z)=z+\sum_{k=2}^{\infty} b_{k} z^{k} .
$$

Let $\mathbb{N}=\{0,1,2, \ldots, n, \ldots\}$.

Definition 1.1. [3] For $f \in \mathcal{A}, \lambda \geq 0$ and $n \in \mathbb{N}$, the operator $\mathscr{D}_{\lambda}^{n}$ is defined by $\mathscr{D}_{\lambda}^{n}: \mathcal{A} \rightarrow \mathcal{A}$

$$
\begin{gathered}
\mathscr{D}_{\lambda}^{0} f(z)=f(z), \\
\mathscr{D}_{\lambda}^{1} f(z)=(1-\lambda) f(z)+\lambda z f^{\prime}(z)=\mathscr{D}_{\lambda} f(z), \ldots \\
\mathscr{D}_{\lambda}^{n+1} f(z)=(1-\lambda) \mathscr{D}_{\lambda}^{n} f(z)+\lambda z\left(\mathscr{D}_{\lambda}^{n} f(z)\right)^{\prime}=\mathscr{D}_{\lambda}\left(\mathscr{D}_{\lambda}^{n} f(z)\right), z \in U
\end{gathered}
$$


Remark 1.2. If $f \in \mathcal{A}$ and $f(z)=z+\sum_{k=2}^{\infty} a_{k} z^{k}$, then

$$
\mathscr{D}_{\lambda}^{n} f(z)=z+\sum_{k=2}^{\infty}[1+(k-1) \lambda]^{n} a_{k} z^{k}, z \in U .
$$

Remark 1.3. For $\lambda=1$ in the above definition we obtain the Sălăgean differential operator [10].

Definition 1.4. [8] For $f \in \mathcal{A}, n \in \mathbb{N}$, the operator $\mathscr{R}^{n}$ is defined by $\mathscr{R}^{n}: \mathcal{A} \rightarrow \mathcal{A}$,

$$
\begin{gathered}
\mathscr{R}^{0} f(z)=f(z), \quad \mathscr{R}^{1} f(z)=z f^{\prime}(z), \ldots \\
(n+1) \mathscr{R}^{n+1} f(z)=z\left(\mathscr{R}^{n} f(z)\right)^{\prime}+n \mathscr{R}^{n} f(z), z \in U .
\end{gathered}
$$

Remark 1.5. If $f \in \mathcal{A}$ and $f(z)=z+\sum_{k=2}^{\infty} a_{k} z^{k}$, then

$$
\mathscr{R}^{n} f(z)=z+\sum_{k=2}^{\infty} \frac{(n+k-1) !}{n !(k-1) !} a_{k} z^{k}, z \in U .
$$

Definition 1.6. [1] Let $\gamma, \lambda \geq 0, n \in \mathbb{N}$. Denote by $\mathscr{L}^{n}$ the operator given by

$$
\mathscr{L}^{n}: \mathcal{A} \rightarrow \mathcal{A}, \quad \mathscr{L}^{n} f(z)=(1-\gamma) \mathscr{R}^{n} f(z)+\gamma \mathscr{D}_{\lambda}^{n} f(z), z \in U .
$$

Remark 1.7. If $f \in \mathcal{A}$ and $f(z)=z+\sum_{k=2}^{\infty} a_{k} z^{k}$, then

$$
\mathscr{L}^{n} f(z)=z+\sum_{k=2}^{\infty}\left\{\gamma[1+(k-1) \lambda]^{n}+(1-\gamma) \frac{(n+k-1) !}{n !(k-1) !}\right\} a_{k} z^{k}, z \in U .
$$

Definition 1.8. [6] Let $f$ and $g$ be analytic functions in $U$. We say that the function $f$ is subordinate to the function $g$, if there exists a function $w$, which is analytic in $U$ and $w(0)=0,|w(z)|<1, z \in U$, such that $f(z)=g(w(z)), \forall z \in U$. We denote by $\prec$ the subordination relation.

Definition 1.9. For $\widetilde{\lambda} \geq 0 ;-1 \leq A<B \leq 1 ; 0<B \leq 1 ; n \in \mathbb{N}$ let $L(n, \tilde{\lambda}, A, B)$ denote the subclass of $\mathcal{A}$ which contain functions $f(z)$ of the form (1.1) such that

$$
(1-\widetilde{\lambda})\left(\mathscr{L}^{n} f(z)\right)^{\prime}+\widetilde{\lambda}\left(\mathscr{L}^{n+1} f(z)\right)^{\prime} \prec \frac{1+A z}{1+B z} .
$$

Attiya and Aouf defined in [4] the class $\mathscr{R}(n, \lambda, A, B)$ with a condition like (1.3), but there instead of the operator $\mathscr{L}^{n}$ they used the Ruscheweyh operator.

Definition 1.10. [12], [9] A function $f(z)$ of the form (1.1) is said to be in the class $V\left(\theta_{k}\right)$ if $f \in \mathcal{A}$ and $\arg \left(a_{k}\right)=\theta_{k}, \forall k \geq 2$. If $\exists \delta \in \mathbb{R}$ such that $\theta_{k}+(k-1) \delta \equiv \pi(\bmod 2 \pi)$, $\forall k \geq 2$ then $f(z)$ is said to be in the class $V\left(\theta_{k}, \delta\right)$. The union of $V\left(\theta_{k}, \delta\right)$ taken over all possible sequences $\left\{\theta_{k}\right\}$ and all possible real numbers $\delta$ is denoted by $V$.

Let $V L(n, \tilde{\lambda}, A, B)$ denote the subclass of $V$ consisting of functions

$$
f(z) \in L(n, \widetilde{\lambda}, A, B) .
$$


Definition 1.11. The modified Hadamard product of two functions $f$ and $g$ of the form (1.1) and (1.2), and which belong to $V\left(\theta_{k}, \delta\right)$ is defined by (see also [5], [9], [11])

$$
(f * g)(z)=z-\sum_{k=2}^{\infty} a_{k} b_{k} z^{k}=(g * f)(z) .
$$

Theorem 1.12. [7] Let the function $f(z)$ defined by (1.1) be in $V$. Then $f(z) \in V L(n, \widetilde{\lambda}, A, B)$, if and only if

$$
\sum_{k=2}^{\infty} k C_{k}(1+B)\left|a_{k}\right| \leq(B-A)
$$

where

$$
C_{k}=\gamma[1+(k-1) \lambda]^{n}[1+\widetilde{\lambda} \lambda(k-1)]+\frac{(n+k-1) !}{n !(k-1) !}(1-\gamma)\left[1+\widetilde{\lambda} \frac{k-1}{n+1}\right]
$$

The extremal functions are:

$$
f(z)=z+\frac{B-A}{k C_{k}(1+B)} e^{i \theta_{k}} z^{k},(k \geq 2) .
$$

\section{Main results}

Theorem 2.1. If $f \in V L\left(n, \tilde{\lambda}, A_{1}, B\right), g \in V L\left(n, \tilde{\lambda}, A_{2}, B\right)$ then

$f * g \in V L\left(n, \tilde{\lambda}, A^{*}, B\right)$, where $A^{*}=B-\frac{\left(B-A_{1}\right)\left(B-A_{2}\right)}{2 C_{2}(1+B)}$.

The result is sharp.

Proof. Let $f \in V L\left(n, \widetilde{\lambda}, A_{1}, B\right), g \in V L\left(n, \tilde{\lambda}, A_{2}, B\right)$ and suppose they have the form (1.1). Since $f \in V L\left(n, \tilde{\lambda}, A_{1}, B\right)$ we have

$$
\frac{\sum_{k=2}^{\infty} k C_{k}(1+B)\left|a_{k}\right|}{B-A_{1}} \leq 1
$$

and for $g \in V L\left(n, \tilde{\lambda}, A_{2}, B\right)$ we have

$$
\frac{\sum_{k=2}^{\infty} k C_{k}(1+B)\left|b_{k}\right|}{B-A_{2}} \leq 1 .
$$

We know from Theorem 1.12 that $f * g \in V L\left(n, \widetilde{\lambda}, A^{*}, B\right)$ if and only if

$$
\frac{\sum_{k=2}^{\infty} k C_{k}(1+B)\left|a_{k} b_{k}\right|}{B-A^{*}} \leq 1 .
$$

By using the Cauchy-Schwarz inequality for (2.1) and (2.2) we have

$$
\frac{\sum_{k=2}^{\infty} k C_{k}(1+B) \sqrt{\left|a_{k} b_{k}\right|}}{\sqrt{\left(B-A_{1}\right)\left(B-A_{2}\right)}} \leq 1 .
$$


We note that

$$
\frac{\sum_{k=2}^{\infty} k C_{k}(1+B)\left|a_{k} b_{k}\right|}{B-A^{*}} \leq \frac{\sum_{k=2}^{\infty} k C_{k}(1+B) \sqrt{\left|a_{k} b_{k}\right|}}{\sqrt{\left(B-A_{1}\right)\left(B-A_{2}\right)}}
$$

implies (2.3). But this is implied by

or

$$
\frac{\left|a_{k} b_{k}\right|}{B-A^{*}} \leq \frac{\sqrt{\left|a_{k} b_{k}\right|}}{\sqrt{\left(B-A_{1}\right)\left(B-A_{2}\right)}}
$$

$$
\sqrt{\left|a_{k} b_{k}\right|} \leq \frac{B-A^{*}}{\sqrt{\left(B-A_{1}\right)\left(B-A_{2}\right)}},(k \geq 2) .
$$

From Theorem 1.12 we have:

$$
\left|a_{k}\right| \leq \frac{B-A_{1}}{k C_{k}(1+B)} \text { and }\left|b_{k}\right| \leq \frac{B-A_{2}}{k C_{k}(1+B)},(k \geq 2)
$$

this implies that

$$
\sqrt{\left|a_{k} b_{k}\right|} \leq \frac{\sqrt{\left(B-A_{1}\right)\left(B-A_{2}\right)}}{k C_{k}(1+B)},(k \geq 2) .
$$

From (2.5) we obtain that (2.4) holds if

$$
\frac{\sqrt{\left(B-A_{1}\right)\left(B-A_{2}\right)}}{k C_{k}(1+B)} \leq \frac{B-A^{*}}{\sqrt{\left(B-A_{1}\right)\left(B-A_{2}\right)}}
$$

or equivalently

$$
A^{*} \leq B-\frac{\left(B-A_{1}\right)\left(B-A_{2}\right)}{k C_{k}(1+B)} .
$$

But $k C_{k}<(k+1) C_{k+1},(k \geq 2)$ so

$$
\begin{gathered}
B-\frac{\left(B-A_{1}\right)\left(B-A_{2}\right)}{k C_{k}(1+B)} \geq B-\frac{\left(B-A_{1}\right)\left(B-A_{2}\right)}{2 C_{2}(1+B)},(k \geq 2) \\
\Rightarrow A^{*}=B-\frac{\left(B-A_{1}\right)\left(B-A_{2}\right)}{2 C_{2}(1+B)} .
\end{gathered}
$$

The result is sharp, because if

$$
\begin{aligned}
& f(z)=z+\frac{B-A_{1}}{2 C_{2}(1+B)} e^{i \theta_{1}} z^{2} \in V L\left(n, \tilde{\lambda}, A_{1}, B\right) \\
& g(z)=z+\frac{B-A_{2}}{2 C_{2}(1+B)} e^{i \theta_{2}} z^{2} \in V L\left(n, \widetilde{\lambda}, A_{2}, B\right)
\end{aligned}
$$

then $f * g \in V L\left(n, \widetilde{\lambda}, A^{*}, B\right)$ and satisfy (1.5) with equality. Indeed,

$$
2 C_{2}(1+B) \frac{\left(B-A_{1}\right)\left(B-A_{2}\right)}{2^{2} C_{2}^{2}(1+B)^{2}}=B-A^{*}
$$

because

$$
B-A^{*}=\frac{\left(B-A_{1}\right)\left(B-A_{2}\right)}{2 C_{2}(1+B)} .
$$


Corollary 2.2. If $f, g \in V L(n, \tilde{\lambda}, A, B)$ then $f * g \in V L\left(n, \tilde{\lambda}, A^{*}, B\right)$, where

$$
A^{*}=B-\frac{(B-A)^{2}}{2 C_{2}(1+B)} \text {. }
$$

The result is sharp.

Theorem 2.3. If $f \in V L\left(n, \tilde{\lambda}, A, B_{1}\right), g \in V L\left(n, \tilde{\lambda}, A, B_{2}\right)$ then $f * g \in V L\left(n, \tilde{\lambda}, A, B^{*}\right)$, where

$$
B^{*}=A+\frac{\left(B_{1}-A\right)\left(B_{2}-A\right)(A+1)}{2 C_{2}\left(1+B_{1}\right)\left(1+B_{2}\right)-\left(B_{1}-A\right)\left(B_{2}-A\right)} .
$$

The result is sharp.

Proof. Let $f \in V L\left(n, \widetilde{\lambda}, A, B_{1}\right), g \in V L\left(n, \widetilde{\lambda}, A, B_{2}\right)$ and suppose they have the form (1.1). Since $f \in V L\left(n, \tilde{\lambda}, A, B_{1}\right)$ we have

$$
\frac{\sum_{k=2}^{\infty} k C_{k}\left(1+B_{1}\right)\left|a_{k}\right|}{B_{1}-A} \leq 1
$$

and for $g \in V L\left(n, \tilde{\lambda}, A, B_{2}\right)$ we have

$$
\frac{\sum_{k=2}^{\infty} k C_{k}\left(1+B_{2}\right)\left|b_{k}\right|}{B_{2}-A} \leq 1 .
$$

We know from Theorem 1.12 that $f * g \in V L\left(n, \widetilde{\lambda}, A, B^{*}\right)$ if and only if

$$
\frac{\sum_{k=2}^{\infty} k C_{k}\left(1+B^{*}\right)\left|a_{k} b_{k}\right|}{B^{*}-A} \leq 1 .
$$

By using the Cauchy-Schwarz inequality for (2.6) and (2.7) we have

$$
\frac{\sum_{k=2}^{\infty} k C_{k} \sqrt{\left(1+B_{1}\right)\left(1+B_{2}\right)} \sqrt{\left|a_{k} b_{k}\right|}}{\sqrt{\left(B_{1}-A\right)\left(B_{2}-A\right)}} \leq 1 .
$$

We note that

$$
\frac{\sum_{k=2}^{\infty} k C_{k}\left(1+B^{*}\right)\left|a_{k} b_{k}\right|}{B^{*}-A} \leq \frac{\sum_{k=2}^{\infty} k C_{k} \sqrt{\left(1+B_{1}\right)\left(1+B_{2}\right)} \sqrt{\left|a_{k} b_{k}\right|}}{\sqrt{\left(B_{1}-A\right)\left(B_{2}-A\right)}}
$$

implies (2.8). But this is implied by

$$
\frac{\left|a_{k} b_{k}\right|\left(1+B^{*}\right)}{B^{*}-A} \leq \frac{\sqrt{\left|a_{k} b_{k}\right|} \sqrt{\left(1+B_{1}\right)\left(1+B_{2}\right)}}{\sqrt{\left(B_{1}-A\right)\left(B_{2}-A\right)}}
$$

or

$$
\sqrt{\left|a_{k} b_{k}\right|} \leq \frac{\left(B^{*}-A\right) \sqrt{\left(1+B_{1}\right)\left(1+B_{2}\right)}}{\left(1+B^{*}\right) \sqrt{\left(B_{1}-A\right)\left(B_{2}-A\right)}},(k \geq 2) .
$$


From Theorem 1.12 we have:

$$
\left|a_{k}\right| \leq \frac{B_{1}-A}{k C_{k}\left(1+B_{1}\right)} \text { and }\left|b_{k}\right| \leq \frac{B_{2}-A}{k C_{k}\left(1+B_{2}\right)},(k \geq 2)
$$

this implies that

$$
\sqrt{\left|a_{k} b_{k}\right|} \leq \frac{\sqrt{\left(B_{1}-A\right)\left(B_{2}-A\right)}}{k C_{k} \sqrt{\left(1+B_{1}\right)\left(1+B_{2}\right)}},(k \geq 2) .
$$

from (2.10) we obtain that (2.9) holds if

$$
\frac{\sqrt{\left(B_{1}-A\right)\left(B_{2}-A\right)}}{k C_{k} \sqrt{\left(1+B_{1}\right)\left(1+B_{2}\right)}} \leq \frac{\left(B^{*}-A\right) \sqrt{\left(1+B_{1}\right)\left(1+B_{2}\right)}}{\left(1+B^{*}\right) \sqrt{\left(B_{1}-A\right)\left(B_{2}-A\right)}}
$$

or equivalently

$$
B^{*} \geq A+\frac{\left(B_{1}-A\right)\left(B_{2}-A\right)(A+1)}{k C_{k}\left(1+B_{1}\right)\left(1+B_{2}\right)-\left(B_{1}-A\right)\left(B_{2}-A\right)} .
$$

But $k C_{k}<(k+1) C_{k+1},(k \geq 2)$ so:

$$
\begin{gathered}
A+\frac{\left(B_{1}-A\right)\left(B_{2}-A\right)(A+1)}{k C_{k}\left(1+B_{1}\right)\left(1+B_{2}\right)-\left(B_{1}-A\right)\left(B_{2}-A\right)} \\
\leq A+\frac{\left(B_{1}-A\right)\left(B_{2}-A\right)(A+1)}{2 C_{2}\left(1+B_{1}\right)\left(1+B_{2}\right)-\left(B_{1}-A\right)\left(B_{2}-A\right)}, \quad(k \geq 2) \\
\Rightarrow B^{*}=A+\frac{\left(B_{1}-A\right)\left(B_{2}-A\right)(A+1)}{2 C_{2}\left(1+B_{1}\right)\left(1+B_{2}\right)-\left(B_{1}-A\right)\left(B_{2}-A\right)} .
\end{gathered}
$$

The result is sharp, because if

$$
\begin{aligned}
& f(z)=z+\frac{B_{1}-A}{2 C_{2}\left(1+B_{1}\right)} e^{i \theta_{1}} z^{2} \in V L\left(n, \tilde{\lambda}, A, B_{1}\right) \\
& g(z)=z+\frac{B_{2}-A}{2 C_{2}\left(1+B_{2}\right)} e^{i \theta_{2}} z^{2} \in V L\left(n, \tilde{\lambda}, A, B_{2}\right)
\end{aligned}
$$

then $f * g \in V L\left(n, \widetilde{\lambda}, A, B^{*}\right)$ and satisfy (1.5) with equality. Indeed,

$$
\left(1+B^{*}\right) 2 C_{2} \frac{\left(B_{1}-A\right)\left(B_{2}-A\right)}{2^{2} C_{2}^{2}\left(1+B_{1}\right)\left(1+B_{2}\right)}=B^{*}-A
$$

because

$$
B^{*}-A=\frac{\left(B_{1}-A\right)\left(B_{2}-A\right)(A+1)}{2 C_{2}\left(1+B_{1}\right)\left(1+B_{2}\right)-\left(B_{1}-A\right)\left(B_{2}-A\right)} .
$$

Corollary 2.4. If $f, g \in V L(n, \tilde{\lambda}, A, B)$ then $f * g \in V L\left(n, \tilde{\lambda}, A, B^{*}\right)$, where

$$
B^{*}=A+\frac{(B-A)^{2}(A+1)}{2 C_{2}(1+B)^{2}-(B-A)^{2}} \text {. }
$$

The result is sharp. 
Theorem 2.5. If $f_{j} \in V L\left(n, \tilde{\lambda}, A_{j}, B\right), \quad j=\overline{1, m}, \quad m \in\{2,3,4, \ldots\}$ then

$$
f_{1} * f_{2} * \ldots * f_{m} \in V L\left(n, \widetilde{\lambda}, A^{(m-1) *}, B\right),
$$

where

$$
A^{(m-1) *}=B-\frac{\prod_{j=1}^{m}\left(B-A_{j}\right)}{2^{m-1} C_{2}^{m-1}(1+B)^{m-1}} .
$$

The result is sharp.

Proof. For the proof we use the mathematical induction method and suppose that $f_{j}, \forall j$ have the form (1.1).

Let $m=2$. If $f_{j} \in V L\left(n, \tilde{\lambda}, A_{j}, B\right), j=\overline{1,2}$ then $f_{1} * f_{2} \in V L\left(n, \tilde{\lambda}, A^{*}, B\right)$ where $A^{*}=B-\frac{\left(B-A_{1}\right)\left(B-A_{2}\right)}{2 C_{2}(1+B)}$, from Theorem 2.1 is true.

Assume that the result is true for $m=k$, that is,

$$
f_{1} * f_{2} * \ldots * f_{k} \in V L\left(n, \tilde{\lambda}, A^{(k-1) *}, B\right)
$$

where $A^{(k-1) *}=B-\frac{\prod_{j=1}^{k}\left(B-A_{j}\right)}{2^{k-1} C_{2}^{k-1}(1+B)^{k-1}}$.

Next, we prove that the result is true for $k+1$ :

then $f_{1} * f_{2} * \ldots * f_{k} * f_{k+1} \in V L\left(n, \tilde{\lambda}, A^{k *}, B\right)$, where

$$
\begin{aligned}
& A^{k *}=B-\frac{\left(B-A^{(k-1) *}\right)\left(B-A_{k+1}\right)}{2 C_{2}(1+B)}
\end{aligned}
$$

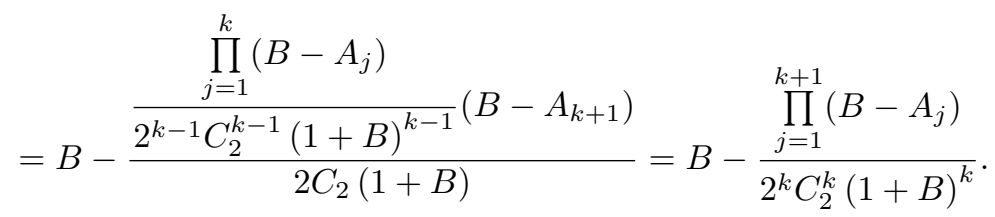

The result is sharp, because if

$$
f_{j}(z)=z+\frac{B-A_{j}}{2 C_{2}(1+B)} e^{i \theta_{j}} z^{2} \in V L\left(n, \tilde{\lambda}, A_{j}, B\right), \quad j=\overline{1, m}
$$

then

$$
f_{1} * f_{2} * \ldots * f_{m}(z)=z+\frac{\prod_{j=1}^{m}\left(B-A_{j}\right)}{2^{m-1} C_{2}^{m-1}(1+B)^{m-1}} e^{i\left(\theta_{1}+\theta_{2}+\ldots+\theta_{m}\right)} z^{2}
$$

satisfy (1.5) with equality. Indeed,

$$
2 C_{2}(1+B) \prod_{j=1}^{m}\left(B-A_{j}\right) \frac{1}{2^{m} C_{2}^{m}(1+B)^{m}}=B-A^{(m-1) *} .
$$


Theorem 2.6. If $f_{j} \in V L\left(n, \tilde{\lambda}, A, B_{j}\right), j=\overline{1, m}, m \in\{2,3,4, \ldots\}$ then

$$
f_{1} * f_{2} * \ldots * f_{m} \in V L\left(n, \tilde{\lambda}, A, B^{(m-1) *}\right),
$$

where

$$
B^{(m-1) *}=A+\frac{(A+1) \prod_{j=1}^{m}\left(B_{j}-A\right)}{2^{m-1} C_{2}^{m-1} \prod_{j=1}^{m}\left(1+B_{j}\right)-\prod_{j=1}^{m}\left(B_{j}-A\right)} .
$$

The result is sharp.

Proof. The proof is similar to the demonstration for Theorem 2.5.

\section{References}

[1] Alb Lupaş, A., Andrei, L., Aspects of univalent holomorphic functions involving Ruscheweyh derivative and generalized Salagean operator, Journal of Computational Analysis \& Applications, 19(2015), no. 1, 272-277.

[2] Al-Amiri, H.S., On Ruscheweyh derivatives, Ann. Polon. Math., 38(1980), 87-94.

[3] Al-Oboudi, F.M. On univalent functions defined by a generalized Salagean operator, Ind. J. Math. Math. Sci., 27(2004), 1429-1436.

[4] Attiya, A.A., Aouf, M.K., A study on certain class of analytic functions defined by Ruscheweyh derivative, Soochow J. Math., 33(2007), no. 2, 273-289.

[5] Hossen, H.M., Sălăgean, G.S., Aouf, M.K., Notes on certain classes of analytic functions with negative coefficients, Mathematica, 39(1997), no. 2, 165-179.

[6] Mocanu, P.T., Bulboacă, T., Sălăgean, G.S., The geometric theory of univalent functions, Casa Cărţii de Ştiinţă, Cluj-Napoca, 2006.

[7] Páll-Szabó, Á.O., Certain class of analytic functions with varying arguments defined by Sălăgean and Ruscheweyh derivative, Mathematica (Cluj), 59(82)(2017), no. 1-2, 80-88.

[8] Ruscheweyh, S., New criteria for univalent functions, Proc. Amer. Math. Soc., 49(1975), 109-115.

[9] Sălăgean, G.S., Convolution properties of some classes of analytic functions with negative coefficients, Proc. Int. Symposium New. Develop. Geometric Function Th. and its Appl. GFTA, Univ. Kebangsaan Malaysia, 10-13 Nov. 2008, 12-16.

[10] Sălăgean, G.S., Subclasses of univalent functions, Lecture Notes in Math., Springer Verlag, 1013(1983), 362-372.

[11] Schild, A., Silverman, H., Convolution of univalent functions with negative coefficients, Ann. Univ. Mariae Curie-Sklodowska Sect. A, 29(1975), 99-107.

[12] Silverman, H., Univalent functions with varying arguments, Houston J. Math., 17(1981), 283-287.

Ágnes Orsolya Páll-Szabó

Babeş-Bolyai University

Faculty of Mathematics and Computer Sciences

Cluj-Napoca, Romania

e-mail: pallszaboagnes@math.ubbcluj .ro 